\title{
SPECTRAL THEOREM FOR UNBOUNDED STRONGLY CONTINUOUS GROUPS ON A HILBERT SPACE
}

\author{
KHRISTO BOYADZHIEV AND RALPH DELAUBENFELS
}

(Communicated by Palle E. T. Jorgensen)

\begin{abstract}
Suppose $B$ is a closed, densely defined linear operator on a Hilbert space and $a>0$. Denote $\{z|| \operatorname{Im}(z) \mid<b\}$ by $H_{b}$.

We show that $B$ has an $H^{\infty}\left(H_{b}\right)$ functional calculus, for all $b>a$, if and only if $i B$ generates a strongly continuous group of operators of exponential type $a$. We obtain specific upper bounds on $\|f(B)\|$, in terms of $\sup \left\{e^{-b|t|}\left\|e^{i t B}\right\| \mid t \in \mathbf{R}\right\}$.

Corollaries include the spectral theorem for closed operators on a Hilbert space and a generalization of a result due to McIntosh relating imaginary powers and $H^{\infty}$ functional calculi.
\end{abstract}

\section{INTRODUCTION}

The spectral theorem for unbounded operators on a Hilbert space asserts an equivalence between an operator $i B$ generating a (uniformly) bounded strongly continuous group and $B$ having a $C_{0}(\mathbf{R})$ functional calculus (this then implies that $B$ is a scalar type spectral operator). In this paper, we show that, on a Hilbert space, $i B$ generating an arbitrary strongly continuous group of bounded operators is equivalent to $B$ having a functional calculus defined for $H^{\infty}$ of a horizontal strip. More specifically, if we write $H_{\phi}$ for $\{z|| \operatorname{Im}(z) \mid<\phi\}, i B$ generates a strongly continuous group of exponential type $\omega$ if and only if $B$ has an $H^{\infty}\left(H_{\phi}\right)$ functional calculus for all $\phi>\omega$. The usual spectral theorem is a corollary of our result.

It is interesting that the holomorphic, or sectoral, analogue of these results is false; that is, there exist generators of bounded strongly continuous holomorphic semigroups on a Hilbert space that do not have $H^{\infty}$ of a sector (where the spectrum of the generator is contained) functional calculi (see $[16,1])$. This would seem to imply that, in some sense, a strongly continuous group is "better" than a strongly continuous holomorphic semigroup, or a strip is better than a sector.

Another corollary of our results in this paper is a generalization of a result due to McIntosh [15]. We show that, on a Hilbert space, $A$ has bounded imaginary powers of exponential type $\theta<\pi / 2$ if and only if $A$ has an $H^{\infty}\left(S_{\phi}\right)$ functional

Received by the editors April 13, 1992.

1991 Mathematics Subject Classification. Primary 47A10, 47A60, 47D05, 47D10.

(C) 1993 American Mathematical Society $0002-9939 / 93 \$ 1.00+\$ .25$ per page 
calculus, $\forall \phi>\theta$, where $S_{\theta} \equiv\{z|| \arg (z) \mid<\theta\}$, without assuming that $A$ is of type $\theta$ (see Definition 1.3).

Some interesting work on $H^{\infty}$ functional calculi appears in $[1,4,6,11$, $15,16,18]$. A property that is weaker than having an $H^{\infty}\left(S_{\theta}\right)$ functional calculus, for some $\theta<\pi$, and is equivalent on a Hilbert space, is having bounded imaginary powers. This property and many interesting applications have been studied recently in $[1,9,10,15,17,19]$. A Banach space analogue of the spectral theorem appears in [2].

In $\S$ II we present some preliminary results on how to construct a functional calculus for $B$, on any Banach space, and what properties this functional calculus has. This is a special case of a sort of unbounded version of the RieszDunford functional calculus that is introduced in [8]. This enables us to define $e^{B}$ explicitly and gets our functional calculus started.

Throughout this paper, all operators are linear on a Banach space $X$. The space $B(X)$ will be all bounded operators from $X$ into itself. We will write $\rho(A)$ for the resolvent set of the operator $A, \sigma(A)$ for the spectrum, $\mathscr{D}(A)$ for the domain, and $\operatorname{Im}(A)$ for the image.

Definition 1.1. Suppose $\mathscr{F}$ is a Banach algebra of complex-valued functions, $A$ is an operator, and there exists complex $\lambda \in \rho(A)$ such that $g_{\lambda}(z) \equiv(\lambda-z)^{-1} \in$ $\mathscr{F}$.

Then $A$ has a $\mathscr{F}$ functional calculus if there exists a continuous algebra homomorphism, $f \mapsto f(A)$, from $\mathscr{F}$ into $B(X)$, such that $\lambda \in \rho(A)$ and $g_{\lambda}(A)=(\lambda-A)^{-1}$ whenever $g_{\lambda} \in \mathscr{F}$.

Definition 1.2. If $\Omega$ is a (possibly unbounded) region in the complex plane, then $H_{0}^{\infty}(\Omega) \equiv H^{\infty}(\Omega) \cap C_{0}(\bar{\Omega})$.

Definitions 1.3. Suppose $A$ is densely defined.

(a) If $0 \leq \theta<\pi, A$ is said to be of type $\theta$ if, $\forall \psi^{\prime}>\theta, \sigma(A) \subseteq \overline{S_{\psi}}$ and $\left\{\left\|z(z-A)^{-1}\right\| \mid z \notin \overline{S_{\psi}}\right\}$ is bounded.

Note that, for $0<\theta<\pi / 2$, this is equivalent to $-A$ generating a bounded strongly continuous holomorphic semigroup of angle $(\pi / 2-\theta)$. The adjective "bounded" means that, $\forall \phi<(\pi / 2-\theta), \exists M_{\phi}$ such that $\left\|e^{-z A}\right\| \leq M_{\phi}, \forall z \in S_{\phi}$.

This class of operators has received a good deal of recent attention in connection with an operator having bounded imaginary powers or, what is stronger in general and equivalent on a Hilbert space, having an $H^{\infty}\left(S_{\psi}\right)$ functional calculus, $\forall \psi>\theta$. Because of the close relationship between this particular functional calculus and being of type $\theta$, and in order to have more streamlined terminology, we make the following definition:

(b) For $0 \leq \theta<\pi$, we will say that $A$ is of super type $\theta$ if, $\forall \pi>\psi>\theta$, $A$ has an $H^{\infty}\left(S_{\psi}\right)$ functional calculus.

We would also like to introduce analogous definitions for generators of strongly continuous groups.

(c) If $\theta \geq 0$, we will say that $B$ is of horizontal type $\theta$ if $i B$ generates a strongly continuous group of exponential type $\theta$; that is, $\forall \psi>\theta, \exists M_{\psi}$ such that $\left\|e^{i s B}\right\| \leq M_{\psi} e^{\psi|s|}, \forall s \in \mathbf{R}$.

We think of type $\theta$, in (a), as sector type $\theta$.

(d) If $\theta \geq 0$, we will say that $B$ is of horizontal super type $\theta$ if $B$ has an $H^{\infty}\left(H_{\psi}\right)$ functionasl calculus, $\forall \psi>\theta$. 
Note that, in (a)-(d), replacing $H^{\infty}$ by $H_{0}^{\infty}$ does not change the definition.

It is not hard to show that, if $A$ is of (horizontal) super type $\theta$, then $A$ is of (horizontal) type $\theta$.

\section{Preliminaries}

Throughout this paper, $C$ will be a bounded, injective operator.

Definition 2.1. A strongly continuous family of bounded operators $\{W(t)\}_{t \geq 0}$ is a $C$-regularized semigroup if $W(0)=C$ and $W(t) W(s)=W(t+s) \bar{C}$, $\forall s, t \geq 0$.

The generator is defined by

$$
A x \equiv C^{-1}\left(\lim _{t \rightarrow 0} W(t) x-C x\right)
$$

with maximal domain; that is, $\mathscr{D}(A)$ equals the set of all $x$ for which the limit exists and is in $\operatorname{Im}(C)$.

The generator is automatically closed.

We think of $W(t)$ as being $e^{t A} C$.

Definition 2.2. The $C$-resolvent set $\rho_{C}(A)$ for $A$ is the set of all complex $\lambda$ such that $(\lambda-A)$ is injective and $\operatorname{Im}(C) \subseteq \operatorname{Im}(\lambda-A)$.

Thus the operator $(\lambda-A)^{-1} C$ is defined everywhere, for $\lambda \in \rho_{C}(A)$, and is bounded, if $A$ is a closed operator.

We will also write $\sigma_{C}(A)$ for the $C$-spectrum of $A$, the complement of $\rho_{C}(A)$.

Definitions $2.3,2.5$, and 2.7 and Lemmas 2.4 and 2.6 are special cases of the construction in [8].

Definition 2.3. Suppose that there exists $\lambda \in \rho_{C}(A)$ such that $g_{\lambda}(z) \equiv$ $(\lambda-z)^{-1} \in \mathscr{F}$. A $C$-regularized $\mathscr{F}$ functional calculus for $A$ is a map $\Gamma$ from $\mathscr{F}$ into $B(X)$ such that $(\Gamma f)(\Gamma g)=(\Gamma(f g)) C, \forall f, g \in \mathscr{F}$, and $\lambda \in \rho_{C}(A)$, with $\Gamma g_{\lambda}=(\lambda-A)^{-1} C, \forall \lambda$ such tht $g_{\lambda} \in \mathscr{F}$.

We think of $\Gamma f$ as being $f(A) C$.

Lemma 2.4. Suppose $B$ is of horizontal type $\theta \geq 0, \phi>\theta$, and $f$ is holomorphic on $H_{\phi}$ and maps $H_{\phi}$ into a left half-plane. Then

$$
W_{f, \lambda}(t) \equiv \int_{\partial H_{\psi}} e^{t f(w)}(w-B)^{-1} \frac{d w}{2 \pi i(\lambda-w)^{2}} \quad(\theta<\psi<\phi)
$$

$\left(\lambda \notin \overline{H_{0}}\right)$ defines an exponentially bounded $(\lambda-B)^{-2}$-regularized semigroup.

Definition 2.5. If $B$ and $f$ are as in Lemma 2.4, then $f(B)$ is defined to be the generator of $\left\{W_{f, \lambda}(t)\right\}_{t \geq 0}$. It is shown in [8] that this is independent of $\lambda$.

Lemma 2.6. Suppose $B$ is of horizontal type $\theta \geq 0$. Let $C \equiv(\lambda-B)^{-2} \quad(\lambda \notin$ $\left.\overline{H_{\theta}}\right)$. The map $f \mapsto f(B)$, where $f$ is as in Lemma 2.4, has the following properties:

(1) $g_{\lambda}(B)=(\lambda-B)^{-1}, \forall \lambda \notin \overline{H_{\theta}}$, where $g_{\lambda}(w) \equiv(\lambda-w)^{-1}$.

(2) When $f(w)$ is $O\left((1+|w|)^{-2}\right), f(B) \in B(X)$ and is given by

$$
f(B)=\int_{\partial H_{\psi}} f(w)(w-B)^{-1} \frac{d w}{w \pi i} .
$$


(3) When $(f g)(w)$ is $O\left((1+|w|)^{-2}\right)$, with $f, g$ mapping into a left halfplane, $\operatorname{Im}(g(B)) \subseteq \mathscr{D}(f(B))$, with $f(B) g(B)=(f g)(B) \in B(X)$.

(4) $\sigma_{C}(f(B)) \subseteq f\left(\overline{H_{\theta}}\right)$.

(5) $\forall \varphi>\theta, B$ has a $C$-regularized $H^{\infty}\left(H_{\phi}\right)$ functional calculus given by $\Gamma f \equiv f(B) C \quad(|\operatorname{Im}(\lambda)|>\phi)$.

(6) $C(\mathscr{D}(f(B)))$ is dense.

Definition 2.7. When $B$ is of horizontal type $\theta, 0 \leq \theta<\pi / 2, e^{B}$ will be $g(B)$, defined by Definition 2.5, where $g(z) \equiv e^{z}$.

That is, if $C \equiv(\lambda-B)^{-2}\left(\lambda \notin \overline{H_{\theta}}\right),-e^{B}$ is defined to be the generator of the bounded $C$-regularized semigroup

$$
W_{\lambda}(t) \equiv \int_{\partial H_{\psi}} e^{-t e^{w}}(w-B)^{-1} \frac{d w}{2 \pi i(\lambda-w)^{2}} \quad\left(\theta<\psi<\frac{\pi}{2}\right) .
$$

By Lemma 2.6, $\sigma_{C}\left(e^{B}\right) \subseteq \overline{S_{\theta}}$, and $C\left(\mathscr{D}\left(e^{B}\right)\right)$ is dense.

Proposition 2.8. Suppose $\operatorname{Im}(C)$ is dense. Then the following are equivalent:

(a) $B$ is closed and has a $C$-regularized $\mathscr{F}$ functional calculus $\Gamma$ such that

$$
\|(\Gamma f) x\| \leq M\|f\|_{\mathscr{F}}\|C x\| \quad \text { and } \quad f(B) C=\Gamma f, \quad \forall x \in X, f \in \mathscr{F} .
$$

(b) $B$ has an $\mathscr{F}$ functional calculus, with $\|f(B)\| \leq M\|f\|_{\mathscr{F}}, \forall f \in \mathscr{F}$.

Proof. The implication (b) $\rightarrow($ a) is clear. For $(\mathrm{a}) \rightarrow(\mathrm{b})$, for $f \in \mathscr{F}, x \in$ $\operatorname{Im}(C)$, define $f(B) x \equiv(\Gamma f) C^{-1} x$. Clearly $\|f(B) x\| \leq M\|f\|_{\mathscr{F}}\|x\|$. Thus, since $\operatorname{Im}(C)$ is dense, $f(B)$ may be extended to a bounded operator on $X$, with the same paper bound on the norm.

Suppose $\lambda \in \rho_{C}(B)$ and $g_{\lambda} \in \mathscr{F}$. To conclude the proof, it is sufficient to show that $\lambda \in \rho(B)$, since it is clear that $f(B) C=\Gamma f, \forall f \in \mathscr{F}$, and $\operatorname{Im}(C)$ is dense.

Since $B$ is closed and $(\lambda-B)$ is injective, it is sufficient to show that $(\lambda-B)$ is surjective. Fix $x \in X$. By our construction, $g_{\lambda}(B)$ is a bounded extension of $(\lambda-B)^{-1}$ from $\operatorname{Im}(C)$ to $X$. Choose a sequence $x_{n} \in \operatorname{Im}(C)$ converging to $x$. Let $y_{n} \equiv(\lambda-B)^{-1} x_{n}$. Then $y_{n} \rightarrow g_{\lambda} x$, and $(\lambda-B) y_{n} \rightarrow x$. Since $(\lambda-B)$ is closed, this implies that $g_{\lambda} x \in \mathscr{D}(B)$ and $(\lambda-B) g_{\lambda} x=x$, as desired.

The following shows how we may switch back and forth between sectors and horizontal strips.

Corollary 2.9. Suppose $B$ is of horizontal type $\theta, 0 \leq \theta<\pi / 2$. Then the following are equivalent if $\theta<\phi<\pi / 2$ :

(a) $B$ has an $H_{0}^{\infty}\left(H_{\phi}\right)$ functional calculus, with $\|f(B)\| \leq M\|f\|_{H_{0}^{\infty}\left(H_{\phi}\right)}$.

(b) $e^{B}$ has an $H_{0}^{\infty}\left(S_{\phi}\right)$ functional calculus, with $\left\|f\left(e^{B}\right)\right\| \leq M\|f\|_{H_{0}^{\infty}\left(S_{\phi}\right)}$.

Proof. Let $C \equiv(\lambda-B)^{-2}\left(\lambda \notin \overline{H_{\theta}}\right)$. By Lemma 2.6(5), $B$ has a $C$-regularized $H_{0}^{\infty}\left(H_{\phi}\right)$ functional calculus. Thus $e^{B}$ has a $C$-regularized $H_{0}^{\infty}\left(S_{\phi}\right)$ functional calculus given by $\Gamma\left(e^{B}\right) \equiv(f \circ g)(B) C \quad\left(g(z) \equiv e^{z}\right)$. 
Thus, by Proposition 2.8 and Lemma 2.6 (a) is equivalent to having

$$
\|f(B) C x\| \leq M\|C x\|\|f\|_{H_{0}^{\infty}\left(H_{\phi}\right)}, \quad \forall f \in H_{0}^{\infty}\left(H_{\phi}\right),
$$

while $(\mathbf{b})$ is equivalent to having

$$
\|(f \circ g)(B) C x\| \leq M\|C x\|\|(f \circ g)\|_{H_{0}^{\infty}\left(H_{\phi}\right)}, \quad \forall f \in H_{0}^{\infty}\left(S_{\phi}\right) .
$$

Since $g\left(H_{\phi}\right)=S_{\phi}$, this concludes the proof.

Lemma 2.10. Suppose $0 \leq \theta<\pi / 2$ and

(1) $\rho_{C}(A) \subseteq \overline{S_{\theta}}$;

(2) $\exists$ a constant $K$ such that

$$
\int_{\mathbf{R}}\left|\left\langle A\left(A^{2}+r^{2}\right)^{-1} C x, C^{*} x^{*}\right\rangle\right| d r \leq K \pi\|C x\|\left\|C^{*} x^{*}\right\|,
$$

$\forall x \in X, x^{*} \in X^{*}$

(3) A has a C-regularized $H_{0}^{\infty}\left(S_{\theta}\right)$ functional calculus $\Gamma$ such that

$$
\left\langle(\Gamma f) x, C^{*} x^{*}\right\rangle=\int_{\mathbf{R}} f(-i r)\left\langle A\left(A^{2}+r^{2}\right)^{-1} C x, C^{*} x^{*}\right\rangle \frac{d r}{\pi},
$$

$\forall x \in X, x^{*} \in X^{*}$

(4) $\operatorname{Im}(C)$ is dense in $X$, and $\operatorname{Im}\left(C^{*}\right)$ is dense in $X^{*}$;

(5) $A$ is closed.

Then there exists an $H_{0}^{\infty}\left(S_{\pi / 2}\right)$ functional calculus for $A$ such that $\|f(A)\| \leq$ $K\|f\|_{\infty}$ and $f(A) C=\Gamma f, \forall f \in H_{0}^{\infty}\left(S_{\pi / 2}\right)$.

Proof. This follows from Proposition 2.8.

Lemma 2.11. Suppose $A=e^{B}$, where $B$ is of horizontal type $\theta, 0 \leq \theta<\pi / 2$, and $C \equiv\left(\lambda_{1}-A\right)^{-1}\left(\lambda_{2}-B\right)^{-2}$ for some $\lambda_{1} \notin \overline{S_{\theta}}, \lambda_{2} \notin \overline{H_{\theta}}$.

Let $f(A) \equiv(f \circ g)(B)$, where $g(z) \equiv e^{z}$. Then

$$
\left\langle f(A) C x, x^{*}\right\rangle=\int_{\mathbf{R}} f(-i r)\left\langle A\left(A^{2}+r^{2}\right)^{-1} C x, x^{*}\right\rangle \frac{d r}{\pi},
$$

$\forall x \in X, x^{*} \in X^{*}, f \in H^{\infty}\left(S_{\phi}\right), \phi>\theta$.

Proof.

$$
\begin{aligned}
2 \pi i\left\langle f(A) C x, x^{*}\right\rangle \\
=\int_{\partial H_{\phi}} \frac{f\left(e^{w}\right)}{\left(\lambda_{1}-e^{w}\right)}\left\langle(w-B)^{-1} x, x^{*}\right\rangle \frac{d w}{\left(\lambda_{2}-w\right)^{2}} \\
=\int_{\partial H_{\phi}}\left(\int_{\mathbf{R}} f(-i r) \frac{e^{w}}{\left(\lambda_{1}-e^{w}\right)}\left(r^{2}+\left(e^{w}\right)^{2}\right)^{-1} \frac{d r}{\pi}\right)\left\langle(w-B)^{-1} x, x^{*}\right\rangle \frac{d w}{\left(\lambda_{2}-w\right)^{2}} \\
=\int_{\mathbf{R}} f(-i r) \int_{\partial H_{\phi}} \frac{e^{w}}{\left(\lambda_{1}-e^{w}\right)}\left(r^{2}+\left(e^{w}\right)^{2}\right)^{-1}\left\langle(w-B)^{-1} x, x^{*}\right\rangle \frac{d w}{2 \pi i\left(\lambda_{2}-w\right)^{2}}(2 i d r) \\
=2 i \int_{\mathbf{R}} f(-i r)\left\langle A\left(r^{2}+A^{2}\right)^{-1} C x, x^{*}\right\rangle d r .
\end{aligned}
$$


Lemma 2.12. Suppose $B$ is of horizontal type $\theta<\pi$. Then

$$
\left(e^{B}\right)^{1 / 2}\left(e^{B}+\omega\right)^{-1} x=\frac{2}{\sqrt{\omega}} \int_{\mathbf{R}} \omega^{-2 i s}\left(e^{2 \pi s}+e^{-2 \pi s}\right)^{-1} e^{2 i s B} x d s,
$$

for $x \in \mathscr{D}\left(B^{2}\right), \omega \notin(-\infty, 0]$.

Proof. Fix $\lambda \notin \overline{H_{\theta}}$ and $\psi$ such that $\theta<\psi<\operatorname{Im}(\lambda)$.

Let $y \equiv(\lambda-B)^{2} x$.

$$
\begin{aligned}
\frac{2}{\sqrt{\omega}} & \int_{\mathbf{R}} \omega^{-2 i s}\left(e^{2 \pi s}+e^{-2 \pi s}\right)^{-1} e^{2 i s B}(\lambda-B)^{-2} y d s \\
& =\frac{2}{\sqrt{\omega}} \int_{\mathbf{R}} \omega^{-2 i s}\left(e^{2 \pi s}+e^{-2 \pi s}\right)^{-1} \int_{H_{\psi}} e^{2 i s w}(w-B)^{-1} y \frac{d w}{2 \pi i(\lambda-w)^{2}} d s \\
& =\int_{H_{\psi}}\left[\frac{2}{\sqrt{\omega}} \int_{\mathbf{R}} \omega^{-2 i s}\left(e^{2 \pi s}+e^{-2 \pi s}\right)^{-1} e^{2 i s w} d s\right](w-B)^{-1} y \frac{d w}{2 \pi i(\lambda-w)^{2}} \\
& =\int_{H_{\psi}}\left[\left(e^{w}\right)^{1 / 2}\left(e^{w}+\omega\right)^{-1}\right](w-B)^{-1} y \frac{d w}{2 \pi i(\lambda-w)^{2}} \\
& =\left(e^{B}\right)^{1 / 2}\left(e^{B}+\omega\right)^{-1}(\lambda-B)^{-2} y=\left(e^{B}\right)^{1 / 2}\left(e^{B}+\omega\right)^{-1} x .
\end{aligned}
$$

Remark 2.13. The integral transform in Lemma 2.12 appears in [5, Corollary 3.5] for what they call the analytic generator of $B$. This helped motivate Lemma 3.1 in the next section. It is natural to conjecture that $e^{B}$ equals the analytic generator of $B$, but the proof of this does not seem to be obvious. It is not hard to show that the analytic generator is an extension of $e^{B}$ restricted to $\mathscr{D}\left(B^{2} e^{B}\right)$; however, this may or may not be a core for $e^{B}$. In general, if an operator $A$ generates a $C$-regularized semigroup and $\operatorname{Im}(C)$ is dense, it is an open question whether $C(\mathscr{D}(A))$ is a core for $A$.

\section{MAIN RESUlTS}

Throughout this section, $X$ will be a Hilbert space.

Lemma 3.1. Suppose $i B$ generates a strongly continuous group, with $\left\|e^{i s B}\right\| \leq$ $M e^{a|s|}$, for some $0 \leq a<\pi / 2$. Then $A \equiv e^{B}$ is as in Lemma 2.10, with

$$
K=8 M^{2} \int_{0}^{\infty} e^{4 a s}\left(e^{2 \pi s}+e^{-2 \pi s}\right)^{-1} d s
$$

$\theta=a, C \equiv\left(\lambda_{1}-A\right)^{-1}\left(\lambda_{2}-B\right)^{-2}$, for some $\lambda_{1} \notin \overline{S_{a}}, \lambda_{2} \notin \overline{H_{a}}$.

Proof. By Lemma 2.6(4), $A$ satisfies Lemma 2.10(1).

For Lemma 2.10(2) choose $\omega=i t, t>0, r=2 \ln t$, and $x \in \mathscr{D}\left(B^{2}\right)$ in Lemma 2.12

$$
\begin{aligned}
& \sqrt{e^{r / 2}} A^{1 / 2}\left(A+i e^{r / 2}\right)^{-1} x \\
& \quad=2 \sqrt{\frac{2 \pi}{i}} \int_{\mathbf{R}} e^{-i s r}\left[e^{\pi s} e^{2 i s B} x\left(e^{2 \pi s}+e^{-2 \pi s}\right)^{-1}\right] \frac{d s}{\sqrt{2 \pi}}
\end{aligned}
$$

since $s \mapsto\left[e^{\pi s} e^{2 i s B} x\left(e^{2 \pi s}+e^{-2 \pi s}\right)^{-1}\right]$ is in $L^{2}(\mathbf{R}, X)$, the Plancherel Theorem 
implies that

$$
\begin{aligned}
\int_{0}^{\infty} & \left\|A^{1 / 2}(A+i t)^{-1} x\right\|^{2} d t \\
= & \int_{\mathbf{R}}\left\|\sqrt{\frac{1}{2} e^{r / 2}} A^{1 / 2}\left(A+i e^{r / 2}\right)^{-1} x\right\|^{2} d r \\
= & \int_{\mathbf{R}} 4 \pi\left\|\left[e^{\pi s} e^{2 i s B} x\left(e^{2 \pi s}+e^{-2 \pi s}\right)^{-1}\right]\right\|^{2} d s \\
\leq & 4 \pi M^{2}\|x\|^{2} \int_{\mathbf{R}} e^{(2 \pi s+4 a|s|)}\left(e^{2 \pi s}+e^{-2 \pi s}\right)^{-2} d s \\
= & 4 \pi M^{2}\|x\|^{2} \int_{-\infty}^{0} e^{(2 \pi s-4 a s)}\left(e^{2 \pi s}+e^{-2 \pi s}\right)^{-2} d s \\
& +\int_{0}^{\infty} e^{(2 \pi s+4 a s)}\left(e^{2 \pi s}+e^{-2 \pi s}\right)^{-2} d s \\
= & 4 \pi M^{2}\|x\|^{2} \int_{0}^{\infty} e^{(-2 \pi s+4 a s)}\left(e^{2 \pi s}+e^{-2 \pi s}\right)^{-2}+e^{(2 \pi s+4 a s)}\left(e^{2 \pi s}+e^{-2 \pi s}\right)^{-2} d s \\
= & 4 \pi M^{2}\|x\|^{2} \int_{0}^{\infty} e^{4 a s}\left(e^{2 \pi s}+e^{-2 \pi s}\right)^{-1} d s .
\end{aligned}
$$

Identically,

$$
\int_{0}^{\infty}\left\|\left(A^{*}\right)^{1 / 2}\left(A^{*}+i t\right)^{-1} x^{*}\right\|^{2} d t \leq 4 \pi M^{2}\|x\|^{2} \int_{0}^{\infty} e^{4 a s}\left(e^{2 \pi s}+e^{-2 \pi s}\right)^{-1} d s,
$$

for $x^{*} \in \mathscr{D}\left(\left(B^{*}\right)^{2}\right)$.

Thus, for $x \in X, x^{*} \in X^{*}$,

$$
\begin{aligned}
& {\left[\int_{\mathbf{R}}\left|\left\langle A\left(A^{2}+t^{2}\right)^{-1} C x, C^{*} x^{*}\right\rangle\right| d t\right]^{2}=\left[2 \int_{0}^{\infty}\left|\left\langle A\left(A^{2}+t^{2}\right)^{-1} C x, C^{*} x^{*}\right\rangle\right| d t\right]^{2}} \\
& =\left[2 \int_{0}^{\infty}\left|\left\langle A^{1 / 2}(A+i t)^{-1} C x,\left(A^{*}\right)^{1 / 2}\left(A^{*}+i t\right)^{-1} C^{*} x^{*}\right\rangle\right| d t\right]^{2} \\
& \leq\left[2 \int_{0}^{\infty}\left\|A^{1 / 2}(A+i t)^{-1} C x\right\|^{2} d t\right]\left[\int_{0}^{\infty}\left\|\left(A^{*}\right)^{1 / 2}\left(A^{*}+i t\right)^{-1} C^{*} x^{*}\right\|^{2} d t\right. \\
& \leq\left(8 \pi M^{2} \int_{0}^{\infty} e^{4 a s}\left(e^{2 \pi s}+e^{-2 \pi s}\right)^{-1} d s\right)^{2}\|C x\|^{2}\left\|C^{*} x^{*}\right\|^{2} .
\end{aligned}
$$

Lemma 2.10(3) now follows from Lemma 2.11.

By Lemma $2.6(6)$, if $C_{1} \equiv\left(\lambda_{2}-B\right)^{-2}$, then $C_{1}(\mathscr{D}(A))$ is dense. But $C_{1}(\mathscr{D}(A))$ is contained in $\operatorname{Im}(C)$, since, for $y \in \mathscr{D}(A), C_{1} y=C\left(\lambda_{1}-A\right) y$. Thus Lemma 2.10(4) is also valid.

The following is a version of our results that states the relationship between the rate of growth of the group and the upper bound on the norm of the functional calculus explicitly. Part (2) is valid on any Banach space.

Theorem 3.2. (1) Suppose $i B$ generates a strongly continuous group, with $\left\|e^{i s B}\right\|$ $\leq M e^{a|s|}$, for some $0 \leq a$. Then $\forall b>a, B$ has an $H_{0}^{\infty}\left(H_{b}\right)$ functional calculus, with

$$
\|f(B)\| \leq K(M, a, b)\|f\|_{H^{\infty}\left(H_{b}\right)},
$$


where

$$
K(M, a, b) \equiv \frac{4 M^{2}}{\pi} \int_{0}^{\infty} \frac{e^{a s / b}}{\left(e^{s}+e^{-s}\right)} d s .
$$

(2) Suppose $B$ has an $H_{0}^{\infty}\left(H_{a}\right)$ functional calculus, with

$$
\|f(B)\| \leq M\|f\|_{H^{\infty}\left(H_{a}\right)} .
$$

Then $i B$ generates a strongly continuous group, with $\left\|e^{i s B}\right\| \leq M e^{a|s|}$.

Proof. For (1), let $\alpha \equiv \pi / 2 b$, so that $\alpha a<\pi / 2$ Lemmas 3.1 and 2.10 and Corollary 2.9 imply that $\alpha B$ has an $H_{0}^{\infty}\left(H_{\pi / 2}\right)$ functional calculus, with

$$
\|f(\alpha B)\| \leq\left[8 \pi M^{2} \int_{0}^{\infty} e^{4 \alpha a s}\left(e^{2 \pi s}+e^{-2 \pi s}\right)^{-1} d s\right]\|f\|_{H^{\infty}\left(H_{\pi / 2}\right)},
$$

for $f \in H_{0}^{\infty}\left(H_{\pi / 2}\right)$. Thus, for $g \in H_{0}^{\infty}\left(H_{\pi / 2 \alpha}\right)$,

$$
\|g(B)\| \leq\left[8 \pi M^{2} \int_{0}^{\infty} e^{4 \alpha a s}\left(e^{2 \pi s}+e^{-2 \pi s}\right)^{-1} d s\right]\|g\|_{H^{\infty}\left(H_{\pi / 2 \alpha}\right)},
$$

and (1) now follows by substituting $\pi / 2 b$ for $\alpha$.

For (2), note that

$$
\left\|(z-B)^{-n}\right\| \leq M /(|\operatorname{Im}(z)|-a)^{n},
$$

$\forall n \in \mathbf{N},|\operatorname{Im}(z)|>\alpha$. By the Hille-Yosida theorem, to show that $i B$ generates a strongly continuous group such that $\left\|e^{i s B}\right\| \leq M e^{a|s|}$, all that remains is to show that $B$ is densely defined. For $x, y \in H$, since the map $f \mapsto\langle f(B) x, y\rangle$ is a bounded linear functional on $H_{0}^{\infty}\left(H_{a}\right), \exists$ a complex-valued measure $\mu$ of bounded variation on $H_{a}$ such that

$$
\langle f(B) x, y\rangle=\int_{H_{\varphi}} f(w) d \mu(w) \quad \forall f \in C_{0}\left(\overline{H_{a}}\right) \cap H^{\infty}\left(H_{a}\right) .
$$

By dominated convergence,

$$
\lim _{t \rightarrow \infty}\left\langle i t(i t+B)^{-1} x, y\right\rangle=\int_{H_{a}} \lim _{t \rightarrow \infty} i t(i t+w)^{-1} d \mu(w)=\int_{H_{a}} d \mu(w)=\langle x, y\rangle \text {. }
$$

Since $(i t+B)^{-1} x \in \mathscr{D}(B), \forall t$, this concludes the proof.

Corollary 3.3 (Spectral Theorem). Suppose iB generates a bounded strongly continuous group. Then $B$ is a scalar-type spectral operator with real spectrum. Proof. Suppose $\left\|e^{i s B}\right\| \leq M, \forall s \in \mathbf{R}$. By Theorem 3.2(1), with $a=0$, for any $g$ holomorphic and converging to zero at infinity, in a horizontal strip containing the real line,

$$
\|g(B)\| \leq M^{2} \sup \{|g(r)| \mid r \in \mathbf{R}\} .
$$

Since such functions are dense in $C_{0}(\mathbf{R})$, this shows that $B$ has a $C_{0}(\mathbf{R})$ functional calculus. Since $X$ is reflexive, this implies that $B$ is a scalar type spectral operator with real spectrum.

Remark 3.4. Note that, when $i B$ generates a group of isometries, Theorem 3.2 implies, as in the proof of Corollary 3.3, that

$$
\|f(B)\| \leq\|f\|_{\infty} \quad \forall f \in C_{0}(\mathbf{R}) .
$$

Even when $B$ is a bounded operator, Theorem 3.2 and Corollary 3.3 are surprising, in that they give much more information than could be obtained from the Riesz-Dunford functional calculus.

In the next theorem, (b) $\rightarrow($ a) is valid in any Banach space. 
Theorem 3.5. The following are equivalent, for any $\omega>0$ :

(a) $B$ is of horizontal type $\omega$.

(b) $B$ is of horizontal super type $\omega$.

Proof. (a) $\rightarrow$ (b) follows from Theorem 3.2(1), and (b) $\rightarrow$ (a) follows from Theorem 3.2(2).

Definition 3.6. We will say that $A$ has a strongly continuous group of bounded imaginary powers if there exists an operator $B$ such that $i B$ generates a strongly continuous group and $A=e^{B}$. Then $A^{i s} \equiv e^{i s B}$.

The following generalizes $[15, \S 8,(\mathrm{~b}) \leftrightarrow(\mathrm{c})]$ by removing the hypothesis that $A$ be of type $\theta$ from (a).

Corollary 3.7. The following are equivalent, if $0<\theta<\pi$;

(a) $A$ has a strongly continuous group of bounded imaginary powers of exponential type $\theta<\pi$.

(b) $A$ is of super type $\theta$.

Proof. Corollary 2.9 and Theorem 3.5.

\section{EXAMPLES}

Example 4.1. For $\varepsilon>0$, let $X \equiv H^{2}\left(H_{\varepsilon}\right)$, the set of all functions $f: \overline{H_{\varepsilon}} \rightarrow$ C holomorphic on $H_{\varepsilon}$ such that the map $x \mapsto f(x \pm i \varepsilon)$ is in $L^{2}(\mathbf{R})$. Let $B f(z) \equiv z f(z)$, with maximal domain; that is, $i B$ generates

$$
e^{i t B} f(z) \equiv e^{i t z} f(z) \quad\left(z \in \overline{H_{\varepsilon}}, t \in \mathbf{R}\right) .
$$

Then $B$ is of horizontal super type $\varepsilon$ and $H_{0}^{\infty}\left(H_{\varepsilon}\right)$ is the largest Banach algebra $\mathscr{F}$ for which $B$ has an $\mathscr{F}$ functional calculus.

Thus, even on a Hilbert space, our results are the best possible for generators of strongly continuous groups that are not uniformly bounded.

Example 4.2. For $1 \leq p<\infty$, let $i B \equiv d / d x$, the generator of translation on $L^{p}(\mathbf{R})$.

For $p=1, e^{B}$ is not of type $\theta<\pi$ (see [14]; here one may show directly with Fourier multiplier theory that the analytic generator is an extension of $e^{B}$ ). Using multiplier theory, it is shown in [6] that

(1) for $1<p<\infty, e^{B}$ is of type zero,

(2) for $p \neq 2, e^{B}$ does not have an $H^{\infty}\left(S_{\theta}\right)$ functional calculus, for any $\theta$ between 0 and $\pi$.

Thus $B$ does not have an $H^{\infty}\left(H_{\theta}\right)$ functional calculus, for $p \neq 2$, although $B$ is of horizontal type zero. Thus the phenomena of this paper appear to be natural only to Hilbert spaces, even for generators of bounded strongly continuous groups.

Example 4.3. Choose a function $\omega$ from $\mathbf{R}$ into $[0, \infty)$ such that

$$
\sup _{x \in \mathbf{R}} \frac{\omega(x+t)}{\omega(x)}<\infty \quad \forall t \in \mathbf{R} \text {. }
$$

For example, $\omega(x) \equiv e^{x}$, but not $\omega(x) \equiv e^{x^{2}}$. Let $X \equiv L^{2}(\mathbf{R}, \omega(x) d x)$; that is,

$$
\langle f, g\rangle \equiv \int_{\mathbf{R}} f(x) \overline{g(x)} \omega(x) d x
$$


Then it may be shown that translation is strongly continuous on $X$. Let $i B$ be the generator. Then by our results, $i B$ is of super horizontal type $\varepsilon$, where

$$
\varepsilon \equiv \sup _{t \in \mathbf{R}} \frac{1}{t} \log \left[\sup _{x \in \mathbf{R}} \frac{\omega(x+t)}{\omega(x)}\right]
$$

\section{REFERENCES}

1. J. B. Baillon and Ph. Clement, Examples of unbounded imaginary powers of operators, J. Funct. Anal. 100 (1991), 419-434.

2. M. Balabane, H. Emamirad, and M. Jazar, Spectral distributions and generalization of Stone's theorem, Math. Z. (to appear).

3. K. Boyadzhiev and R. deLaubenfels, $H^{\infty}$-functional calculus for perturbations of generators of holomorphic semigroups, Houston J. Math. 17 (1991), 131-147.

4. - Semigroups and resolvents of bounded variation, imaginary powers and $H^{\infty}$ functional calculus, Semigroup Forum 45 (1992), 372-384.

5. I. Cioranescu and L. Zsiảo, Analytic generators for one-parameter groups, Tôhoku Math. J. 28 (1976), 327-362.

6. M. Cowling, I. Doust, A. McIntosh, and A. Yagi, Banach space operators with an $H^{\infty}$ functional calculus, in preparation.

7. E. B. Davies, One-parameter semigroups, Academic Press, London, 1980.

8. R. deLaubenfels, Unbounded holomorphic functional calculus and abstract Cauchy problems for operators with polynomially bounded resolvents, J. Funct. Anal. (to appear).

9. G. Dore and A. Venni, On the closedness of the sum of two closed operators, Math. Z. 196 (1987), 189-201.

10. __ Some results about complex powers of closed operators, J. Math. Anal. Appl. 149 (1990), 124-136.

11. X. T. Duong, $H^{\infty}$ functional calculus of elliptic operators with $C^{\infty}$ coefficients on $L^{p}$ spaces of smooth domains, J. Austral. Math. Soc. Ser. A 48 (1990), 113-123.

12. $\longrightarrow, H_{\infty}$ functional calculus of second order elliptic PDE on $L^{p}$ spaces, Miniconference on Operators in Analysis, Proc. Center Math. Anal. Austral. Nat. Univ., vol. 24, Austral. Nat. Univ., Canberra, 1989, pp. 91-102.

13. J. A. Goldstein, Semigroups of operators and applications, Oxford, New York, 1985.

14. E. Marschall, On the analytic generator of a group of operators, Indiana Univ. Math. J. 35 (1986), 289-309.

15. A. McIntosh, Operators which have an $H^{\infty}$ functional calculus, Miniconference on Operator Theory and PDE, Proc. Center Math. Anal. Austral. Nat. Univ., vol. 14, Austral. Nat. Univ., Canberra, 1986, pp. 210-231.

16. A. McIntosh and A. Yagi, Operators of type $\omega$ without a bounded $H^{\infty}$-functional calculus, Miniconference on Operators in Analysis, Proc. Center Math. Anal. Austral. Nat. Univ., vol. 24, Austral. Nat. Univ., Canberra, 1989.

17. J. Prüss and H. Sohr, On operators with bounded imaginary powers in Banach spaces, Math. Z. 203 (1990), 429-452.

18. W. Ricker, Spectral properties of the Laplace operator in $L^{p}(\mathbf{R})$, Osaka J. Math. 25 (1988), 399-410.

19. A. Yagi, Applications of the purely imaginary powers of operators in Hilbert spaces, J. Funct. Anal. 73 (1987), 216-231.

Department of Mathematics, Ohio Northern University, Ada, OHio 45810

E-mail address: boyadz@onu.edu

Department of Mathematics, Ohio University, Athens, Ohio 45701 